\title{
CLEM with Commercially Available Reagents to Facilitate Immunolocalization
}

\author{
Leslie Cummins, ${ }^{1,5 *}$ Vincent Tu, ${ }^{3}$ Joshua Mayoral, ${ }^{3}$ Louis M. Weiss, ${ }^{3,4}$ Frank P. Macaluso, ${ }^{1,2,5}$ \\ and Vera DesMarais ${ }^{1,2,5}$ \\ ${ }^{1}$ Analytical Imaging Facility, Albert Einstein College of Medicine, Bronx, NY \\ ${ }^{2}$ Department of Anatomy \& Structural Biology, Albert Einstein College of Medicine, Bronx, NY \\ ${ }^{3}$ Department of Pathology Albert Einstein College of Medicine, Bronx, NY \\ ${ }^{4}$ Department of Medicine, Albert Einstein College of Medicine, Bronx, NY \\ ${ }^{5}$ Gruss-Lipper Biophotonics Center, Albert Einstein College of Medicine, Bronx, NY \\ *leslie.gunther@einsteinmed.org
}

\begin{abstract}
There is often a need to locate the same cellular structure of interest in light and electron microscopy, which can be a difficult task. Here we present a method that uses only commercially available reagents and standard epi-fluorescence and transmission electron microscopy (TEM) technology to make correlative light and electron microscopy (CLEM) available to a large group of researchers without specialized CLEM hardware. This was achieved by seeding cells on photo-etched gridded cover slips and staining the protein to be localized with a secondary antibody coupled to both a fluorophore and $10 \mathrm{~nm}$ gold. The presence of the grid allowed for the alignment of light microscopy images with TEM images and the double-labeled antibody revealed co-localization of the fluorophore with gold particles.
\end{abstract}

Keywords: transmission electron microscopy, immunoelectron microscopy, fluorescence microscopy, correlative microscopy, Toxoplasma gondii

\section{Introduction}

In order to examine cellular localization of a protein of interest with a particular biological structure, it is common to utilize primary antibodies against the protein with subsequent fluorophore conjugated secondary antibody staining and image acquisition with fluorescence microscopy. When the morphology of cellular structures needs to be investigated at high resolution, transmission electron microscopy (TEM) can be used. However, at times there is a need to investigate the same cell or cellular structure with both fluorescence microscopy and TEM to visualize protein localization with high-resolution morphology. This can be achieved by combining fluorescence microscopy with TEM by using correlative light and electron microscopy (CLEM). While there are many high-end CLEM techniques available from a variety of different manufacturers that streamline the process, these often require the purchase of expensive specialized equipment $[1,2]$. We developed an approach to CLEM using only commercially available reagents and standard epi-fluorescence and TEM systems. This makes CLEM universally available to researchers who have access to regular fluorescence microscopes and TEMs, but not the specialized equipment typically used to facilitate CLEM. To address these issues, we developed a method to prepare and fix samples on photo-etched, gridded cover slips stained first with a primary and then a secondary antibody conjugated to both an Alexa fluorophore and $10 \mathrm{~nm}$ gold. This allowed observation of identical fields of view in both fluorescence and TEM systems with a single staining procedure.
The model system to which we applied this technique was a monolayer of human fibroblasts infected with an Apicomplexan intracellular parasite Toxoplasma gondii [3]. We were interested in the biology and localization of a $T$. gondii bradyzoite-specific protein, termed MAG2, that we had identified in proteomic studies of the cyst wall of T. gondii [4]. Toxoplasma gondii, an Apicomplexan parasite, can cause significant disease in both immune competent and immunocompromised humans. The predilection of this parasite for the central nervous system causing necrotizing encephalitis and for the eye causing chorioretinitis constitutes its major threat to patients. The development of these diseases is a consequence of the transition of bradyzoites, found within tissue cysts (for example, in the CNS within neurons), into actively replicating tachyzoites. Tissue cysts, therefore, serve as a reservoir for the reactivation of toxoplasmosis when the host becomes immuno-compromised with conditions such as AIDS or organ transplantation, or immune-compromised due to impaired immune responses to the pathogen when the infection has been acquired in utero. Understanding T. gondii developmental biology and formation of the cyst wall will provide the necessary underpinning for control strategies such as vaccine development, as well as the development of therapeutic agents that could eliminate latency and prevent reactivation toxoplasmosis.

\section{Materials and Methods}

Bright-field and fluorescence image acquisition Human fibroblast cells infected with Toxoplasma gondii (Pru strain [1]) were grown on photo-etched cover slips (EMS \#72264-23), fixed with $4 \%$ buffered paraformaldehyde, and immunolabeled for MAG2 (TgME49_209755) [4] using murine monoclonal antibody 20C3 [4] as the primary antibody and Alexa $488 / 10 \mathrm{~nm}$ colloidal gold goat anti-mouse IgG (Invitrogen/ Fisher Scientific \#A31561) as the secondary antibody. Images were acquired on a DeltaVision Personal Epifluorescence Microscope (Applied Precision-GE Healthcare). One set of images was acquired with a $20 \times$ NA 0.3 air objective and another set with a $60 \times$ NA 1.42 oil objective, both in phase contrast and green fluorescence (excitation filter 470/40 and emission filter 525/50). At 60×, fluorescent images were acquired as $\mathrm{Z}$-stacks (step size of $0.2 \mu \mathrm{m}$ ) and deconvolved using SoftWorx (GE Healthcare) software.

TEM image acquisition After imaging of Alexa 488 labeled MAG2, the cells were fixed with $2.5 \%$ glutaraldehyde 
in $0.1 \mathrm{M}$ sodium cacodylate buffer for 30 minutes, rinsed with $0.1 \mathrm{M}$ sodium cacodylate buffer, post-fixed with $1 \%$ osmium tetroxide in $0.1 \mathrm{M}$ sodium cacodylate for 30 minutes, rinsed with buffer, and then rinsed with water. Cells were then en bloc stained with 2\% uranyl acetate (aqueous), dehydrated through a graded series of ethanol $(30,50,70,95,100,100 \%)$, followed by propylene oxide and infiltrated with LX112 epoxy resin (Ladd Research Industries, Burlington, VT). The regions of interest (ROIs) imaged with fluorescence were located using a light microscope, and BEEM capsules were inverted over these areas. Samples were polymerized at $60^{\circ} \mathrm{C}$ for 60 hours, and blocks were popped off the cover slip. The general region of interest (ROI) was cut out and remounted on a flat BEEM capsule for sectioning. Trimming to the specific ROI was done using a Trimtool $45^{\circ}$ (Diatome), and blocks were then serial thin sectioned $(70 \mathrm{~nm})$ en face on a Leica UC7 ultramicrotome using a Diatome Ultra $35^{\circ} \mathrm{knife}$. Sections were collected on formvar-coated slot grids and stained with uranyl acetate and lead citrate. Imaging was performed on a JEOL 1200EX TEM, and micrographs were recorded using Kodak 4489 film.

\section{Results}

Cells were grown on photo-etched, gridded cover slips with alpha-numeric coordinates to facilitate relocating the same cells of interest within a grid. T. gondii cells selected for fluorescent imaging contained toxoplasma vacuoles that were well labeled for MAG2 protein located in the matrix of the toxoplasma cysts [5]. Fluorescence and bright-field (phase contrast) images were acquired on a standard epi-fluorescence microscope. First, images were acquired at low magnification (20× objective) to show an overview of the area of interest, including a number and a letter (see Figure 1A, overlay of brightfield and fluorescent image acquired with a $20 \times$ objective with the number 8 and letter $\mathrm{M}$ of the etched grid clearly visible). Then, a smaller area of that field of view was imaged again as a $\mathrm{z}$-stack in fluorescence and brightfield (phase contrast) using a $60 \times$ objective (this subsection is indicated as the black square in Figure 1A).

Subsequently, the cells were fixed with glutaraldehyde, post-fixed with osmium tetroxide, processed for electron microscopy using a standard protocol, and serially thin-sectioned. Due to the presence of the alpha-numeric grid, we were able to relocate the same cells by using the corresponding $20 \times$ magnification fluorescent/bright-field images and the gaps left in the sections from the etching on the cover slips. This is demonstrated in Figure $1 \mathrm{~B}$, which shows an early serial section of the fluorescent/bright-field sample shown in $1 \mathrm{~A}$. Arrow heads in A and B indicate the location of the corner of the etched grid and the bottom of the etched number 8 . Once the general area of interest had been identified, we were able to find the cells in subsequent sections in the TEM that corresponded to the cells present in the $20 \times$ magnification fluorescent/brightfield image (Figure 1C).

After high-resolution images of cells were acquired on the TEM, we generated overlays of the $60 \times$ magnification fluorescence/bright-field images and the TEM images of the same cells (Figure 2). The morphology of the parasites is clearly visible in the bright-field image (Figure 2A) as well as the TEM image (Figure 2C). To align the images, we stacked the images in Adobe Photoshop and used the borders of the parasites in brightfield and TEM as a guide for alignment. The fluorescence image (Figure 2B) was passively aligned along with the brightfield image. In addition, we also had to move through the $\mathrm{z}$-dimension of the fluorescence/bright-field $\mathrm{z}$-stack using NIH ImageJ to select a single slice fluorescence/bright-field image that showed the best alignment of the morphologies with the TEM image.

In order to show the gold labeling more clearly, we selected two areas in Figure 2 (see box for areas of selection) and show these at higher magnification in Figures 3 and 4. Specific gold label could be seen on structures between the parasites. There was no gold outside of the cells and very little within the parasites. The gold label and the fluorescent label correlate well, allowing us to conclude that the electron-dense material between the parasites is the MAG2 protein that is labeled by
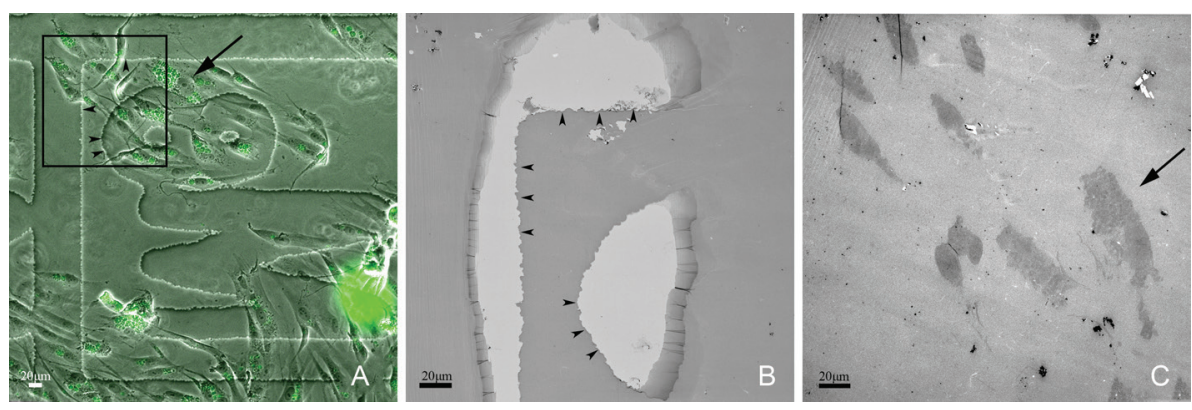

Figure 1: Images required for correlation of light and electron microscopy. Phase/fluorescence overlay (A) showing the markings on the etched cover slips and the location of the cell of interest (arrow). Boxed area is shown in (B) as a TEM image; arrowheads indicate alignment of cover glass etching in first serial section. Low-magnification TEM image $(\mathrm{C})$ of the cells, including cell of interest (arrow) in a subsequent serial thin section. Scale bar indicates $20 \mu \mathrm{m}$.
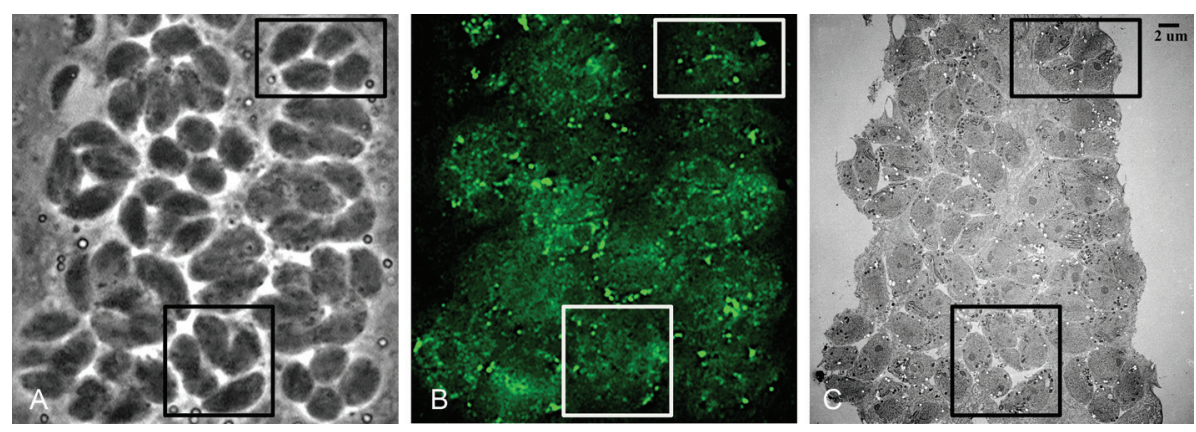

Figure 2: Phase (A), fluorescence (B), and TEM (C) images of the same cell containing multiple parasitophorous vacuoles of Toxoplasma gondii. Boxes show areas of interest further detailed in Figures 3 and 4 . Scale bar shown in $(\mathrm{C})$ indicates $2 \mu \mathrm{m}$ and applies to all panels. 

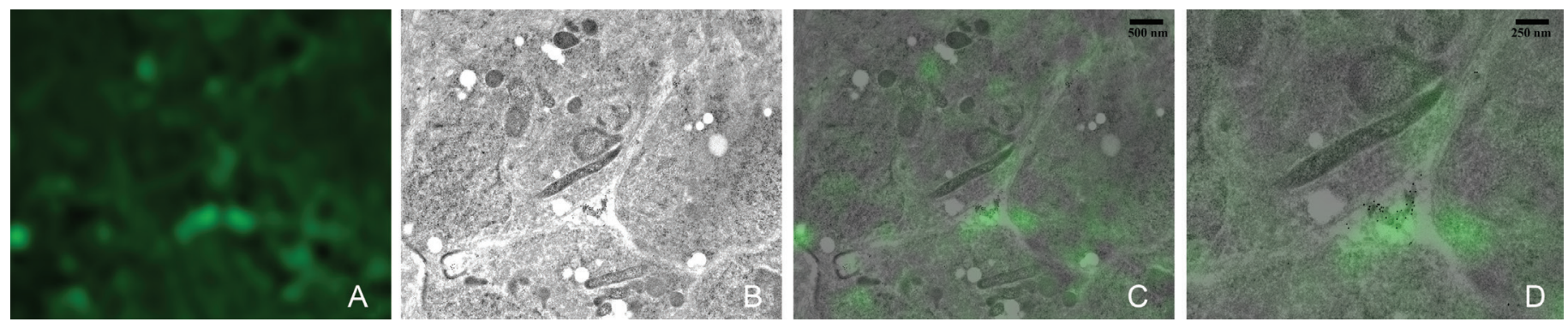

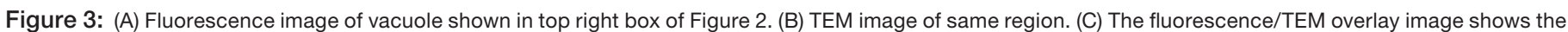

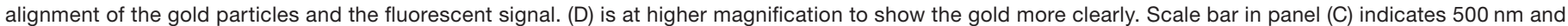
applies to panels (A-C). Scale bar in panel (D) indicates $250 \mathrm{~nm}$.
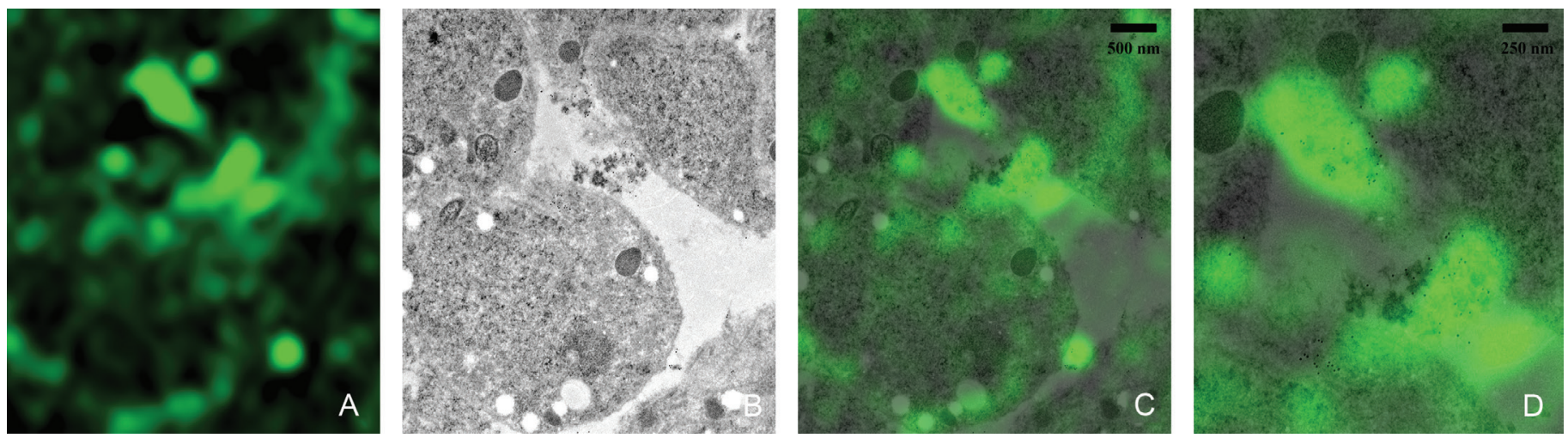

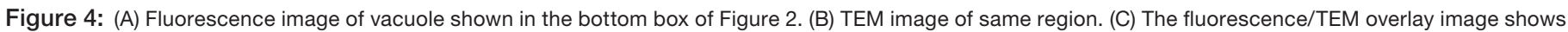

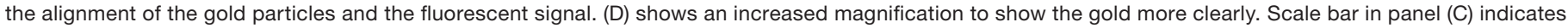
$500 \mathrm{~nm}$ and applies to panels (A-C). Scale bar in panel (D) indicates $250 \mathrm{~nm}$.
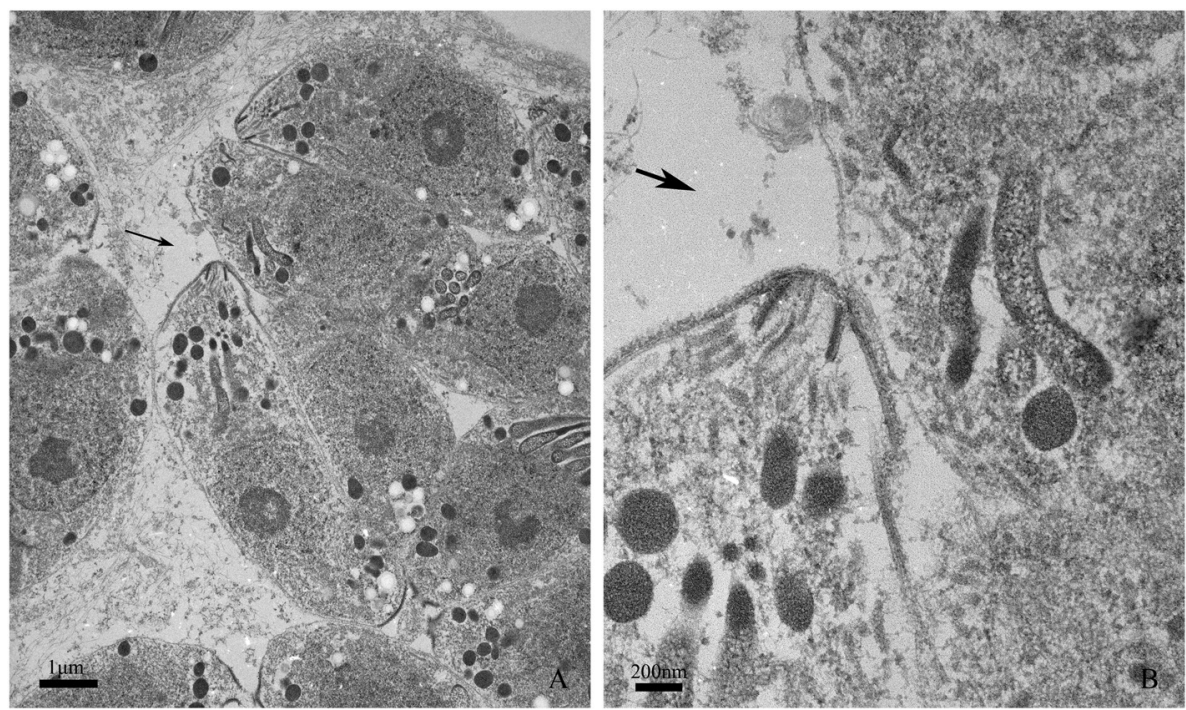

As a negative control cells treated without primary antibody showed no fluorescence nor any gold label (Figure 5) even on the electron dense material within the vacuolar space within the parasitophorous vacuole.

\section{Discussion}

This protocol was developed to facilitate CLEM immunolabeling experiments for a large population of researchers that have access to standard light microscopes and TEMs but no highly specialized CLEM hardware that has been developed by a variety of companies over the last few years. The key to this method is the use of a dual-labeled, commercially available secondary antibody, which is conjugated to both an Alexa 488 fluorophore and

Figure 5: TEM images of no primary sample showing electron dense matrix material (arrow) without gold label at low magnification (A) and high magnification (B). Scale bar in (A) indicates $1 \mu \mathrm{m}$. Scale bar in (B) indicates $200 \mathrm{~nm}$.

$10 \mathrm{~nm}$ gold. Use of this dual-labeled antibody ensures that any fluorescent signal and gold label observed is due to labeling by the same agent. The other key compo-

monoclonal antibody 20C3. Due to the disparity in the section thicknesses, $70 \mathrm{~nm}$ physical in TEM and $200 \mathrm{~nm}$ optical in fluorescence, there are areas where there is fluorescence but no gold. We observed gold in adjacent serial sections of these areas (data not shown). nent of this method is the use of the photo-etched cover glass, which allows the researcher to find the same field of view/ROI in both fluorescence/bright-field images and TEM images, enabling a fast correlation of the two modalities. Although requiring technical expertise in en face thin sectioning and 
serial sectioning, this method enables the researcher to easily use both fluorescence and immuno-gold labeling to identify an antigen of interest in the specific cell of interest without investing in expensive CLEM technical equipment. Another advantage of the method is that using one labeling protocol reduces time and sample preparation.

\section{Conclusions}

In this paper we show that it is possible to conduct CLEM experiments without specialized CLEM hardware simply by taking advantage of commercially available materials, including a double-labeled secondary antibody (Alexa 488 and $10 \mathrm{~nm}$ gold) and a photo-etched cover glass.

\section{Acknowledgments}

This work was done at the Analytical Imaging Facility of Albert Einstein College of Medicine. The work was supported by a NCI cancer center support grant P30CA013330 and a Shared Instrumentation Grant \#1S10OD016214-01A1. Special thanks to Xheni Nishku for Adobe InDesign and Photoshop assistance.

\section{References}

[1] FJ Timmermans and C Otto, Rev Sci Instrum 86(1) (2015) 011501.

[2] K Koistinen et al., EC Microbiology 4(5) (2016) 787-800.

[3] V Tu et al., mBio (2020) doi: 10.1128/mBio.02699-19.

[4] LM Weiss and K Kim, eds., Toxoplasma gondii: The Model Apicomplexan. Perspectives and Methods, Academic Press, Cambridge, MA, 2007.

[5] V Tu et al., mSphere (2020) doi: 10.1128/mSphere.00100-20.

\section{Refurbishing or servicing an electron microscope? Scope us out for your ion pump needs.}

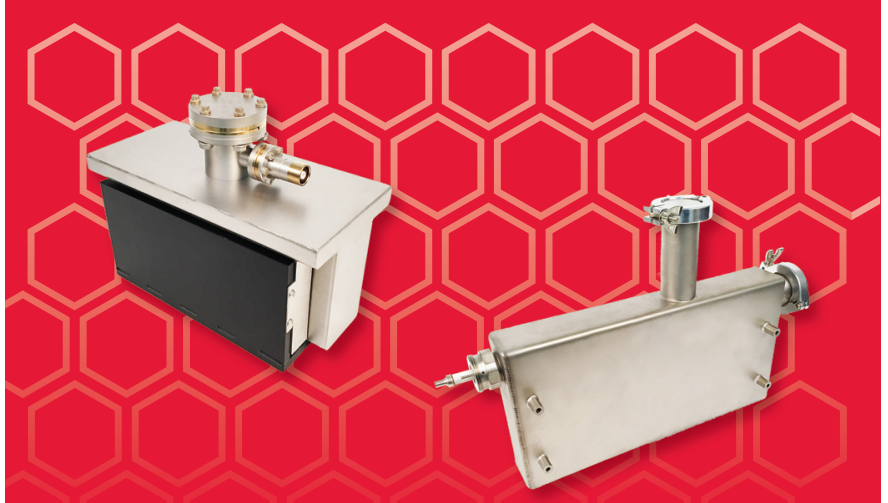

New | Rebuilt | Rebuilding Services

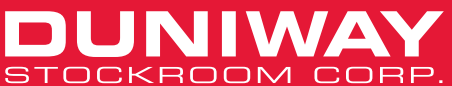

800.448 .8811

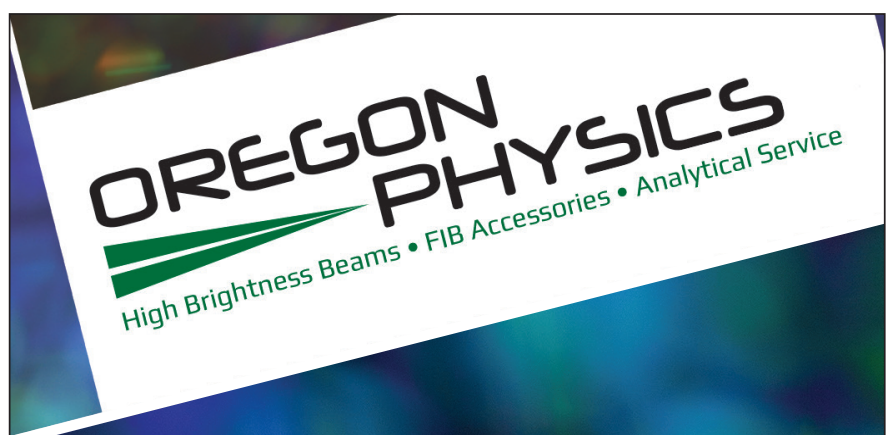

Reduce Your FIB Operating Costs

Managing multiple tools means maintaining system uptime while also saving costs. Let us help with replacement aperture strips, extractors and suppressors.

Oregon Physics has been making FIB consumable parts for over 12 years. Our parts meet and even exceed OEM quality standards so you can order with confidence.

Aperture strips can be made to standard or custom specifications.

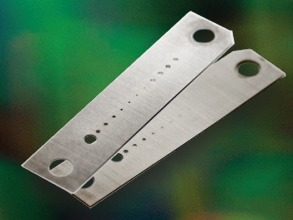

\begin{tabular}{|c|c|}
\hline $\begin{array}{l}\text { For FEI/Thermo Fisher } \\
\text { Scientific FIB Columns: }\end{array}$ & Discounts for: \\
\hline Phoenix & \multirow{5}{*}{$\begin{array}{l}\text { - } 10 \text { or more each } \\
\text { - } 5 \text { or more part sets } \\
\text { - Increased discounts } \\
\text { start at } 20 \text { units. }\end{array}$} \\
\hline Tomahawk & \\
\hline Sidewinder & \\
\hline Magnum & \\
\hline Pre-Lens & \\
\hline
\end{tabular}

We offer regularly scheduled deliveries and fast turnaround for urgent requests.

Contact us to arrange a consumables replacement program to best fit your needs.

+15036010041

info@oregon-physics.com

www.oregon-physics.com 